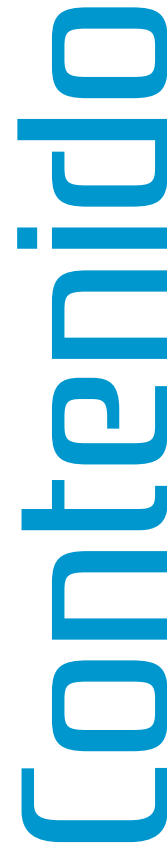

Presentación

Estilos de liderazgo de los directivos escolares costarricenses: transformando las organizaciones educativas

José Antonio García-Martínez \& Virginia Cerdas-Montano

El aprendizaje de la investigación participativa en torno

a la memoria histórica comunitaria.

Ronald Martínez-Villarreal

Teaching Strategies Based on Learning Styles: Promoting Communicative

Competence in Speaking Skills.

Priscilla Carranza-Marchena

Aportes de la escuela en casa para niños y niñas con

necesidades educativas especiales.

Sofía Chirino-Fernández

Reflexiones metodológicas para el análisis de la gestión e indicadores de calidad

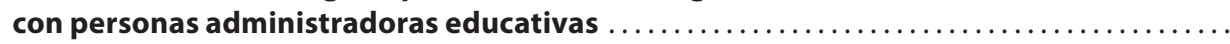

Melissa Mora-Pineda, Adrián Solano-Castro \& Carlos Vargas-Loáiciga

Retórica y educación: una propuesta interpretativa para la Retórica de Aristóteles......

Octavio García-Aguilar

Aportes del Neuromarketing para la atención de personas adultas mayores en centros educativos.

Nancy Vásquez-Flores

Evaluación sumativa para la resolución de problemas en el área de Geometría

Marianela Zumbado-Castro

TIC dirigidas a la superación de barreras educativas de las personas con discapacidad

Alexia Arrieta-Casasola 


\section{COMITÉ EDITORIAL}

\section{Directora}

Jensy Campos Céspedes

Universidad Estatal a Distancia. Costa Rica.

\section{Editor}

Gilbert Ulloa Brenes

Universidad Estatal a Distancia. Costa Rica.

\section{Consejo Editorial}

\section{Adrián Solano Castro}

Universidad Estatal a Distancia. Costa Rica.

Andrea Rojas Vargas

Universidad Estatal a Distancia. Costa Rica.

Rosberly Rojas Campos

Universidad Estatal a Distancia. Costa Rica.

Yarith Rivera Sánchez

Universidad Estatal a Distancia. Costa Rica.

Viviana Berrocal Carvajal

Universidad Estatal a Distancia. Costa Rica.

\section{Consejo Científico Nacional}

Carlos Vargas Loáiciga

COLYPRO, Costa Rica.

Francisco Romero Estrada

Universidad de Costa Rica. Costa Rica.

Gilberto Alfaro Varela

Universidad de Costa Rica. Costa Rica.

Julieta Solórzano Salas

Universidad de Costa Rica. Costa Rica.

Luis Ángel López Ruiz

Universidad de Costa Rica. Costa Rica.

Mónica Arias Monge

Universidad de Costa Rica. Costa Rica.

Rafael Jiménez Corrales

Universidad Nacional, Costa Rica.

Rocío Ramírez González

COLYPRO, Costa Rica.

\section{Consejo Científico Internacional}

Antonio Bartolomé Pina

Universitat de Barcelona. España.

Antonio Nolberto De Oliveira Xavier

Universidade Estadual de Santa Cruz. Brasil.

Bartolomé Chinchilla Chinchilla

Universidad Pedagógica Nacional Francisco Morazán. Honduras.
Bernardo Trejos Murillo

I-Shou University, Taiwán, Provincia de China.

Claudia Martínez Olhagaray

Universidad de la República. Uruguay.

Dulce Fátima Camacho Gutiérrez

Instituto Tecnológico de Monterrey. México.

Edith Soria Valencia

Pontificia Universidad Católica, Perú.

Elena Barberà Gregori

Universitat Oberta de Catalunya. España.

Emiliano Diez Villoria

Universidad de Salamanca. España.

Facundo Corvalán

Universidad Nacional de Rosario. Argentina.

Gina Garcés Ruíz

Universidad Especializada de las Américas. Panamá.

Karl Steffens

Universidad de Colonia. Alemania.

Leonel Carlini López

Universidad Tecnológica Nacional. Argentina.

Luz Díaz Subieta

Universidad Católica Luis Amigó. Colombia.

Manuel Rosales Almendra

Universidad de Guadalajara. México.

Norberto Fernández Lamarra Universidad Nacional de Tres de Febrero. Argentina.

Paloma Antón Ares

Universidad Complutense de Madrid. España.

Raúl Vela Sosa

Universidad Autónoma de Yucatán. México.

Rogelio Ruiz Ríos Universidad Autónoma de Baja California. México.

Sara Fiallos Varela

Universidad Nacional Autónoma de Honduras. Honduras.

Sixto Moya Herrera Investigación y Estudios Educativos y Sindicales de la Actualidad. México.

Teresa Ribeiro Pessoa Universidade de Coimbra. Portugal.

Víctor Torres Covarrubias

Universidad Autónoma de Nayarit. México.

Yolanda Camacho González

Universidad Autónoma de Nayarit. México.

Innovaciones Educativas es una publicación académica semestral de la Universidad Estatal a Distancia (UNED). Las opiniones en la revista no son necesariamente avaladas por la UNED. La responsabilidad compete a los autores. Es una revista de acceso abierto, y se ampara bajo una Licencia Creative Commons Atribución-NoComercial 4.0 Internacional. En tal sentido, se autoriza la reproducción total o parcial de los artículos siempre y cuando se reconozca la autoría de la obra y se cite correctamente la fuente; por tal motivo, debe añadirse además el enlace URL de la revista. Consulte las normas editoriales en el portal de la revista. Servicios de información:

\section{CONTACTO}

Revista Innovaciones Educativas, Escuela de Ciencias de la Educación, Sede Central, Edificio C, Piso 2. Universidad Estatal a Distancia, San José, Costa Rica • Teléfono: (506) 2527-2427 • Correo electrónico: innoveducativas@uned.ac.cr

Para envíos consultar las normas editoriales en la siguiente dirección electrónica: http://investiga.uned.ac.cr/innovacioneseducativas 


\section{Editorial}

Con suma satisfacción, una vez más logramos reunir una selección de trabajos que presentamos ante ustedes, con el fin de que sean de gran provecho en sus propios procesos de desarrollo académico y en los espacios pedagógicos que desarrollan en diferentes contextos educativos. En este nuevo número de nuestra revista, contamos, en primer lugar, con el aporte de José Antonio García Martínez y Virginia Cerdas Montano, quienes nos hablan de los estilos de liderazgo del personal que dirige centros escolares costarricenses y nos recomiendan el liderazgo transformacional como eje dinamizador de la dimensión pedagógica, para el fortalecimiento de procesos identitarios y el logro de metas conjuntas como parte de la calidad educativa. Por su parte, Ronald Martínez Villarreal nos ofrece un artículo sobre el aprendizaje de la investigación participativa en torno a la memoria histórica comunitaria. En este, nos informa sobre procesos de mediación pedagógica en dos experiencias de formación de equipos de gestores comunitarios que investigan la memoria histórica comunitaria mediante el uso de fuentes orales.

En otro orden temático, Priscilla Carranza Marchena aporta al conocimiento sobre la promoción de la competencia comunicativa en la habilidad oral, a través de estrategias de enseñanza basadas en los estilos de aprendizaje propuestos desde la Teoría del Aprendizaje Experimental. Siguiendo con el desarrollo de estrategias pedagógicas innovadoras, Sofía Chirino Fernández nos ofrece un artículo en el que muestra algunos de los aportes que la modalidad de "escuela en casa" proporciona en los procesos educativos de infantes con necesidades educativas especiales. Posteriormente, encontramos el trabajo reflexivo sobre la gestión e indicadores de calidad con administradores educativos que Melissa Mora Pineda, Adrián Solano Castro y Carlos Vargas Loáiciga nos proporcionan desde un proceso de investigación desarrollado por un equipo constituido por personal de la Universidad Estatal a Distancia de Costa Rica y el Colegio de Licenciados y Profesores en Letras, Filosofía, Ciencias y Artes (Colypro).

Desde una perspectiva reflexiva también, Octavio García Aguilar nos propone algunas refrescantes ideas en torno a una nueva propuesta interpretativa para la Retórica de Aristóteles, en la que se plantea que esta obra contiene esbozos de una teoría de la educación y un carácter educativo.

Sobre un tema distinto, pero interesante también, Nancy Vázquez Flores aporta con un ensayo en el que se discuten algunos aportes del Neuromarketing a la atención de personas adultas mayores en centros educativos que trabajan con esta población, especialmente por el énfasis en los procesos neurológicos y cognitivos que se requiere favorecer en ella.

Seguidamente, Marianela Zumbado Castro ofrece los resultados de una revisión bibliográfica acerca de la evaluación sumativa en los procesos de resolución 
de problemas en el área de geometría y nos acerca a lo que la comunidad científica internacional ha venido desarrollando en los últimos años para validar diferentes estrategias e instrumentos de evaluación de los aprendizajes en esa área específica del conocimiento.

Finalmente, encontramos otra revisión de literatura elaborada por Alexia Arrieta Casasola sobre tecnologías de la información y la comunicación, para superar barreras educativas de las personas con discapacidad.

Esperamos que esta selección de trabajos abonen sustantivamente a los procesos de gestión de conocimiento y al mejoramiento de la calidad del campo educativo.

Jensy Campos-Céspedes

Directora de la revista Innovaciones Educativas y del Centro de Investigaciones en Educación, UNED, Costa Rica. ycampos@uned.ac.cr 